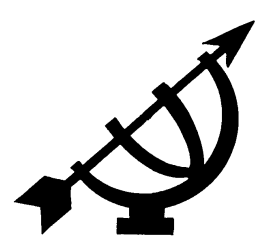

\title{
Research in business ethics
}

\author{
G.J. Rossouw \\ Philosophy Department \\ Rand Afrikaans University \\ JOHANNESBURG \\ E-mail: gjr@lw.rau.ac.za
}

\begin{abstract}
Research in business ethics

Research in business ethics is vital for the expansion and credibility of this fairly recent academic field. This article argues that there are three factors that are crucially important for research in business ethics. They are (a) the ontological assumptions that research in this field is premised upon, (b) the choice of research strategies and methodologies and (c) the role of theory in research practice. Each of these three factors is explored and pitfalls relating to each of them are identified. The article also suggests guidelines for dealing with each of these factors in business ethics research.
\end{abstract}

\section{Introduction}

There are at least three reasons why research in the field of business ethics is vital for the further development of the field.

- Firstly, research should extend the theoretical basis of business ethics. Without such an expanding theoretical basis business ethics will remain an infantile academic field incapable of developing systematic knowledge of its field of study. Explanatory and evaluative theories that will yield a more sophisticated understanding and evaluation of the ethical dimension of economic activity need to be produced.

- Secondly, the academic status of business ethics hinges to a large extent on its ability to produce research that can withstand rigorous academic scrutiny. Only research that complies with the highest academic standards will ensure that business ethics finds its rightful place among other respected and well-established fields of study. 
- Thirdly, the credibility of business ethics as an academic endeavour depends largely on whether research can provide the intellectual tools for practitioners to understand and manage the ethical dimensions of economic activity. Research needs to provide the evidence that the ethical dimensions of business are important and that something can be done about it in order to gain and maintain the support of the actors who deal with the ethical dimension of economic activity (cf. Brigley, 1995a:221). Without such a conviction among the end-users of business ethics the current surge in interest in business ethics is destined to wane.

Given these considerations the question arises as to what should be done to ensure that business ethics research would indeed fulfil these three expectations? My survey of recent literature reveals that there are primarily three aspects of research in business ethics that are particularly important to determine whether these expectations will materialise or not: the ontology of business ethics research, the appropriateness of research strategies and methodologies and the role of theory in research. Each of these three aspects will be explored in this article ${ }^{1}$ in order to develop frameworks and guidelines regarding them.

\section{Ontology in business ethics research}

According to the Cambridge Dictionary of Philosophy (1995:489) ontology refers to views about the nature, constitution and structure of reality or being. Ontology thus conveys the convictions or implied assumptions that one makes about reality - i.e. the way in which one presumes what reality is. All research is based upon ontological assumptions and business ethics is no exception to the rule. Also in business ethics research certain assumptions about the nature of the reality that is studied are made - either explicitly or implicitly. These ontological assumptions determine not only what is researched, but also how research is designed, conducted and presented.

The pitfall that needs to be avoided in business ethics research is onesided (or restrictive) ontologies. Such restrictive ontologies can cause epistemological blind spots that prevent one from seeing certain dimensions of this field of study. This calls for ontologies that will be encompassing enough to capture the variety of ethical dimensions and aspects associated with economic activity.

1 This article forms part of a wider project on "The development of business ethics as academic field in Africa" that was made possible through a Commonwealth Research Fellowship at the University of Cambridge. 
In business ethics research ontological assumptions are inevitably made about the following:

- The economic setting within which ethical behaviour occurs.

- The moral agents whose ethical behaviour is studied within this economic setting.

- Furthermore ontological assumptions are made about the relation between moral agents and the economic setting within which they operate.

The ontological considerations relevant to each of these three aspects of business ethics will now be explored.

\subsection{Economic setting}

Ontological assumptions about the economic setting within which ethical behaviour occur can range from materialistic to idealistic ones. A materialistic ontology regards the economic domain as an objective spatio-temporal phenomenon with its own internal regularities and social laws. Consequently it is assumed that it can be studied in a similar fashion to other material phenomena (cf. Collier, 1995:8; Crane, 1999: 238). Extreme materialist ontologies tend to be closely aligned to empiricist and positivistic approaches to research. It typically shows a preference for precisely formulated theories and quantitative data and will also show an affinity for behaviourist doctrines. 2 The other side of this spectrum is occupied by idealist ontologies. In contrast to the objective nature of materialist ontologies, there is a distinctive subjective ring to idealist ontologies. Idealist ontologies emphasise that the economic domain is shaped by human ideas. The reality shaped by these ideas is not a fixed one, but is continually subjected to processes of reinterpretation. There is no fixed meaning to it, as the meaning of this economic setting is always in a process of flux and renegotiation. Consequently the only way of understanding this economic setting is by way of the interpretations that human beings make of it (cf. Werhane, 1999:51, 65-66). Such idealistic ontologies give preference to hermeneutic research strategies in which symbolic communication within the economic domain is investigated through qualitative methodologies (cf. Collier, 1995:8; Crane, 1999:239).

2 An analysis by Crane (1999) indicates that quantitative research rooted in the positivist tradition is dominating research in business ethics. According to that study $81 \%$ of empirical studies rely on survey data. 
Both extreme materialist and idealist ontologies are restrictive. Materialist ontologies tend to neglect the variety of meanings that individuals can ascribe to institutions, processes and behaviour. It also misses the subtleties imbedded in language as the research instruments associated with it often depend on singular meanings of terms that cannot accommodate the plurality of meanings embedded in language. Furthermore its preference for social and behavioural doctrines makes it hard to grasp contingency and autonomy in human behaviour.

Likewise idealist ontologies also restrict business ethics research. Its preoccupation with symbolic communication is at the cost of understanding the empirical and quantitative dimensions of the economic setting within which ethical behaviour is played out. Its radical openness to new meanings that can be bestowed upon phenomena and behaviour tends to overlook the regularities and patterns that can be discerned within the economic setting, as well as the pressures exerted by this economic setting on individuals to conform to dominant practices. Furthermore its subjectivist nature undermines the development of systematic theories about its domain of study.

The above exposition of the nature of materialist and idealistic ontologies and their respective restrictions makes it imperative to avoid the excesses associated with both of these kinds of ontologies. Instead a more realist ontology of the economic setting in which ethical behaviour occur should be able to accommodate both the valid contributions of materialist and idealist ontologies without restricting research to either of them. It should thus be able to account for both the subjective and objective dimensions in this domain of study. It should be capable of discerning regularities and patterns without losing sight of contingency and human autonomy. What is required are ontological assumptions that allow for a systematic understanding and mapping of the economic setting within which ethical behaviour occurs, while simultaneously allowing for the openness and unpredictability that is typical of human behaviour. Such an ontology will be encompassing enough to accommodate a wide range of research strategies and methodologies suitable for investigating both the objective and subjective dimensions of the economic setting of business ethics.

\subsection{Moral agency}

In the same way that researchers' understanding of the nature of the economic setting in which ethical behaviour occurs influences their approaches to research, so their understanding of the moral agents operating within this setting equally impacts on the way in which they approach research. The major distinction that is relevant to ontological 
assumptions about moral agency is the one between individual and corporate agency. Ontology premised upon the former will only regard individuals as moral agents, while ontology premised upon the latter will regard business organisations as moral agents in their own right. Taken to their respective extreme positions, these two understandings of moral agency create a dilemma: Should only individuals be held morally responsible for the performance of business while organisations are exonerated from moral responsibility? Or should organisations be held morally responsible for their actions and not the individuals who are employed by the organisations?

Defenders of an extreme individual ontology deny that moral responsibility can be ascribed to business organisations (cf. Ladd, 1984). Only biological human beings can, according to their understanding, be expected to adhere to moral standards. The nature of business organisations is such that they pursue purely economic goals. Managers of businesses are obliged to find the most cost-effective means for attaining organisational goals irrespective of moral considerations. Moral considerations are only taken into account when it has a bearing on the costeffectiveness of the organisation's operations. In that sense organisations do not have an inherent moral obligation and are consequently excluded from the category of moral agents. Only individuals qualify for inclusion into the category of moral agents.

Defenders of an extreme corporate ontology believe that individuals can not be held morally accountable for all actions of business organisations. According to them corporations have their own goals, values, policies and decision-making procedures. Individuals employed by corporations are expected to take decisions within the corporation in accordance with these corporate guidelines. They are thus required to subordinate their own goals or values to that of the organisation. Given the complexity of corporate decision-making and the variety of players involved therein, the final decision can not be ascribed to any specific individual, but can only be regarded as a corporate decision (cf. French, 1979). It is thus the corporation that decides to act in a certain way and not a specific individual or even a collection of individuals. Consequently the corporation should be held morally responsible for its actions and not the individuals who participate in the decision-making process.

Both these extreme positions should once more be avoided in research as they can obscure certain aspects of business ethical behaviour. Extreme individual ontologies fail to recognise the capacity of organisations to conduct their affairs with moral sensitivity, whilst extreme corporate ontologies fail to acknowledge the capacity of 
individuals to stand up to organisational directives and to assert their moral autonomy.

It is therefore imperative to avoid these extreme positions in research and to opt for an understanding of moral agency that can accommodate the moral responsibility of individuals and organisations alike. It should be sufficiently flexible to give credit to the ability of individuals to act with moral responsibility despite the pressures exerted upon them by corporate goals, values, policies and procedures. It should, however, also be able to give credit to the ability of organisations to reflect on their goals and intentions, and above all to foresee the moral implications of their decisions (cf. Meyers, 1993; Bishop, 1991).

\subsection{Economic setting and moral agency}

A further vital part of the ontology of business ethics research relates to the understanding of the nature of the interaction between moral agents and the economic setting within which they perform their moral agency. The dividing line between ontologies in this respect is the distinction between personal and cultural understandings of that interaction.

Cultural ontologies will emphasise the social and institutional context within which moral agency is situated. It will see ethical behaviour as embedded in an organisational and societal context characterised by corporate goals, values and processes. In its extreme forms it will portray individual ethical behaviour as a reflex of the cultural context within which it occurs. Such cultural ontologies will promote research on how this cultural setting permeate the ethical behaviour of those involved in it. It will attempt to uncover the impact of among others corporate culture, various stakeholders, professional affiliations, the external environment and the macro-economic context on ethical behaviour (cf. Brigley, 1995b: 22).

Personal ontologies, on the contrary, will emphasise the role of individual moral agency in economic settings. The emphasis will be on the beliefs and convictions that sway individuals in their moral decision-making and behaviour. Rather than focusing on cultural and contextual factors that might have an impact on moral behaviour the focus of research will be on the moral attitudes or values that influence individuals in their decisionmaking. Such personal ontologies result in research that focuses on the attitudes and moral convictions of individuals in situations that require moral decision-making.

Once more both cultural and personal ontologies have the capacity to reveal and to conceal. Cultural ontologies will make researchers aware of 
the impact of organisational, as well as the wider social culture on moral behaviour. It can, however, simultaneously turn a blind eye to those personal dimensions of moral agency that has the capacity of deviating from the general flow of organisational and social culture. Neither will it be particularly apt for dealing with the phenomenon of moral dissidence within organisations. Similarly, personal ontologies can reveal how individuals make their moral decisions, but at the same time it stands in danger of concealing the impact of cultural influences on the moral behaviour of individuals. In this way it might have a blind spot for the gap that often exists between personal moral convictions and actual moral behaviour in organisational settings (cf. Cowton, 1998:424; Robertson, 1993:587). This makes it imperative to utilise ontologies that are capable of accommodating both the personal and cultural understandings of moral agency within organisations (cf. Brigley, 1995b:17-19; Collier, 1995:9).

\subsection{Guidelines for ontology in research}

As ontology determines what researchers see and research, and also how they approach, conduct and report their research, it is imperative that business ethics research should be guided by ontologies encompassing enough to accommodate all the dimensions of ethical agency in the economic settings discussed above. A lack of self-awareness about the ontological assumptions informing and guiding research, is obviously one of the factors that impacts negatively on the quality of research in this field. A more conscious articulation of the ontology on which research is based will go a long way in remedying this deficiency in business ethics research that is by now well documented.

Ontological awareness or sensitivity does not mean that all research should start with an extended discussion of a comprehensive ontology of business ethics. It does, however, require that researchers should be able to indicate the ontological assumptions that underlie their specific research project. In this way they will signal to the research community what contribution their research wish to make to the field of business ethics, whilst simultaneously indicating those dimensions of their object of study that they choose not to investigate. Such intellectual honesty and modesty will reduce the risk of research being labelled simplistic or naïve. 


\section{Strategy and methodology}

Research is a game of justification ${ }^{3}$. Not only do researchers have to justify their findings, but each and every decision in the process of research should equally be justified. This includes the selection of research strategies and methodologies. It is of the utmost importance that the strategy and methodology selected should be appropriate and justifiable for the topic of research. Rigidity with regard to strategy or methodology is destined to undermine the quality of business ethics research.

It is therefore imperative that the ontological flexibility discussed above should be complemented with similar flexibility with regard to the selection of research strategy and methodology. Sensitivity for the potential as well as the handicaps of research methodologies and strategies is in this respect of vital importance. In this section four considerations that should guide decisions on research strategy and methodology in business ethics will be discussed: embeddedness, stage of development, ambiguity and sensitivity in business ethics research.

\subsection{Embeddedness}

The above discussion on the ontology of business ethics research revealed that research in this field is always encapsulated in a tension between freedom and constraint. Ethics in economic activity is always an embedded ethics - embedded in the institutions, frameworks and organisations of economic activity. With regard to our understanding of the economic setting of business ethics it was shown that research ontology should both allow for the objective regularities and patterns that emerge in economic behaviour, but also for the contingency caused by human subjectivity. In discussing moral agency it was pointed out that as moral actors our autonomy is always constrained by conditions beyond our control. When it came to moral agency within economic settings it was equally made clear that our moral agency is always embedded in a cultural context. Thus the embeddedness of business ethics is evident. Moral behaviour within the field of business ethics can never be studied in isolation of the economic setting within which it originates.

This embeddedness of business ethics has important implications for the selection of research strategies and methodologies. Strategies that ignore the economic context that both enables and constrains ethical

See Botes' (2000:180-187) exposition of the role of justification in micro- and macroarguments in research. 
behaviour are bound to produce superficial and not very useful knowledge. What is needed are strategies and methodologies that will be capable of studying ethical behaviour in such a way that not only the freedom of actors (subjectivity and personal autonomy) is understood, but also the impact of the economic setting (objective and cultural) on it.

This ontological consideration indicates what kind of research strategies and methodologies will not suffice in business ethics. Quantitative research, for example, that relies on self-report survey methodologies might be very useful in determining the moral attitudes and convictions of individuals, but it is not particularly helpful in understanding how they actually would behave within the economic setting. ${ }^{4} \mathrm{~A}$ number of factors within the organisational context, such as corporate culture, rolegoverned responsibilities, stakeholder pressure and the like can impact on and even short-circuit these ethical intentions. This makes it imperative to opt for strategies and methodologies that will enable researchers also to study actual moral behaviour and not merely intended behaviour. In order to achieve this, phenomenological research strategies conducted within naturalistic settings is required. A variety of more qualitative research methodologies can be utilised in this regard, such as case studies, narratives, observation, personal in-depth interviews and focus groups. Document analysis of company reports and communications can also be used to uncover how ethical behaviour is institutionalised within the organisational setting (cf. Brigley, 1995b:22 and 1995a:222). Thus, in order to give due credit to the cultural embeddedness of ethical behaviour in business, not only a variety of research strategies is required, but also a variety of research methodologies.

\subsection{Stage of development}

Recently developed fields of study typically suffer certain growth pains that more mature fields have already outgrown. Among these growth pains count an insufficient theoretical basis and confusion about the meaning of key concepts. The requirements for what counts as appropriate research within such recently developed fields have often also not been settled. That business ethics is still such recent field of study, especially on a world-wide scale, is beyond dispute. Its stage of

$4 \quad$ Bain (1995:13-15) argues that the popularity of self-report survey methodologies has more to do with the pressure to publish than with the appropriateness or usefulness thereof. According to his analysis of published research in business ethics it is easier to get published if your research is empirical rather than theoretical, or quantitative rather than qualitative. Also see Cowton (1998:424), Crane (1999:237) and Robertson (1993:587) on the gap that might exist between ethical attitudes and actual ethical behaviour. 
development is an important factor that must be reckoned with in the design of research.

As a recent field of study a lot of effort need to be put into generating new theories and expanding on those that already exist (cf. Crane, 1999:239). Research should therefore be exploratory in order to uncover new dimensions of this field, which can aid the process of theorybuilding. In such an early stage, hypothesis-generating research is usually more desirable and more appropriate than hypothesis-testing research. Such exploratory research is mostly of a qualitative nature and thrives on qualitative rather than quantitative methodologies. The priority that is currently given to quantitative hypothesis-testing research in business ethics is therefore not serving the developmental requirements of the field. This claim is further substantiated by the widely heard complaint that despite the quantity of research activity in the field the theoretical basis of the field is neither expanding satisfactorily nor at a satisfactory rate.

\subsection{Ambiguity}

The ambiguity of key terms in business ethics also has implications for decisions about research strategy and methodology. This ambiguity stems from two sources. The first one has to do with the stage of development of the field. As a recent and developing field there is still not sufficient consensus on the meaning of key terms in its community of researchers. This is something that one can expect to be remedied over time. There is, however, another source of ambiguity that time most probably will not remedy, and that has to do with the very nature of ethics (cf. McDonald \& Donleavy, 1995:845). The contents of key ethical concepts is destined to remain ambiguous, because concepts like good, ethical, right and wrong do not refer to factual states of affairs, but depend on value judgements that people make. Two persons can observe the same occurrence (fact) but end off with totally opposing judgements (value) of the goodness or rightness of what has occurred. The typical moral dilemmas of our time all testify to this reality. If one accepts Maclntyre's (1985) diagnosis of moral dissensus, namely that these differences in value judgements can be related to differences in the ultimate values to which individuals adhere, it is evident that time will not cure this ambiguity associated with ethical judgement. There is no need to regard this as disconcerting. It is par for the course of ethics - and also par for the course of business ethics. Aristotle already admitted this when he said that we should not expect to speak with "the same degree of precision in all our discussions, [...] for it is the mark of the trained mind never to expect more precision in the treatment of any subject than the nature of that subject permits" (Aristotle, 1976:65). Some subjects, 
like business ethics simply do no allow the same precision as others, but that does not imply that we cannot talk in reason about them.

This ambiguity associated with ethics should be accounted for in research strategy and methodology. What is highly problematical is research that pretends that such ambiguity does not exist. When such typically ambiguous key concepts are operationalised in research instruments that do not allow respondents the opportunity to bring their own understandings of these concepts into play, the research results most probably will not be very revealing. Such a conceptual straightjacket (Crane, 1999:241) is likely to tell us more about the pre-judgements of the researchers than about those being researched. What is needed to accommodate these concerns about ambiguity in business ethics research are methodologies that will allow respondents the opportunity to co-construct the meaning of such ambiguous terms (cf. Brigley, 1995b:19). Open-ended questions, personal interviews, focus groups and other methodologies that allow respondents to interact with the researcher are much more suitable for this purpose than close-ended questions, where the only possibility for interaction with the researcher is a tick in a box.

This ambiguity in business ethics research also points to the importance of analysing the communication that occur within organisations, because these subtle differences in meaning are conveyed in linguistic form. Analysis of communication (formal or informal, documented or undocumented) through, for example, content analysis, can be a useful research instrument to uncover the meanings that moral agents within organisations attach to key terms related to ethical behaviour.

\subsection{Sensitivity}

A final consideration that should inform research design has to do with the sensitivity of this area of inquiry (cf. Cowton \& Crisp, 1998:101). Ethical issues are sensitive issues because they reflect on the quality of our being and they can also have detrimental consequences for us. To be praised as someone who acts ethically (with honesty, integrity and care) is something that most people would value, while being labelled as exercising unethical behaviour (that is being dishonest, unscrupulous, cruel) is something to be avoided. Being implicated in unethical behaviour can also be detrimental to one's professional career or can even cost one one's job.

It is therefore not surprising that business ethics research is haunted by the phenomenon of social desirability response bias as well as by a high rate of non-response. Social desirability response bias refers to the 
phenomenon that respondents do not report their actual moral behaviour, but rather how they would like others (including the researcher) to perceive them. Non-response (or refusal to participate in research) is a general phenomenon in research, but it tends to be unacceptably high in business ethics research. 5 It is exactly the sensitive nature of ethics alluded to above that is suspected to be the cause of this high rate of non-response.

Research design in business ethics needs to find ways of dealing and minimising these problems associated with sensitivity. Merely assuring respondents that their anonymity will be ensured is not enough. More creative ways of dealing with it should be designed. These measures can be both of a qualitative and quantitative nature. Qualitative measures for overcoming social desirability response bias includes the use of interpretative research methodologies such as in-depth interviews and focus group interviews (cf. Vyakarnam, 1995:28) that will award researchers the opportunity of probing deeper into the initial reactions of respondents. Naturalistic observation and case studies might also be useful in this regard. In quantitative research randomised response techniques can be used to overcome this form of bias (Robertson, 1993:591). Another approach that might be useful is to use secondary data and secondary research, in which the initial objective was not intended to research ethical issues. By "eavesdropping"6 on these data, access can be gained to information that was not initially intended to serve as ethics research. Thus it is safe to assume that social desirability response bias would not have affected the original data to the same extent. In general, given this sensitive nature of business ethics research, triangulation of methodologies should be the rule rather that the exception (cf. Crane, 1999:242).

The methods suggested above could also go a long way in overcoming non-response, as the main cause of non-response in business ethics research is also suspected to be the sensitivity issue. Whenever high levels of non-response occur, the non-response should not merely be reported but it should itself become a topic of inquiry (cf. Cowton, 1998: 423; Randall \& Gibson, 1990:465).

5 Randall and Gibson (1990:464) argue that given the sensitive nature of business ethics research a response rate of around $70 \%$ is required for findings to be generalisable.

6 Cowton (1998:427) uses this term to indicate that in secondary research it is as if one enters into a discourse between others without them being aware of your presence. 
The justification of research strategy and methodology with respect to the above four considerations can promote the quality of research activity in the field of business ethics. It can also contribute towards the reliability and justifiability of research findings in this field of study.

\section{The role of theory in research}

The relationship between theory and research practice is ideally presented as one where existing theory informs new research and where new research expands on existing theory. The relationship is thus regarded as a mutually enriching and mutually stimulating one. That this ideal has not yet realised in business ethics research is widely lamented (cf. Crane, 1999:239; Nicholson, 1994:581; Robertson, 1993:589; Randall \& Gibson, 1990:461). Research design often neglects to consult existing theory, and research findings are seldom used to extend or build on existing theories. Both these matters are of vital importance for the development of business ethics as academic field.

One of the prerequisites for the development of an academic field is that its theoretical basis should grow in width and depth. The way in which it happens is through research. That does not, however, mean that any research activity will result in the expansion of the theoretical basis of a field of study. It is possible to have a large quantity of research, without it contributing to or improving the quality of the theoretical basis of the field.7 In order for research to be able to contribute to the theoretical basis of the field, new research must deliberately take its point of departure in the existing theoretical basis. By doing that, new research is anchored in what has been achieved thus far and consequently will have a view of what needs to be done in order to improve on what is currently available. Research done in this way has the character of an ongoing discourse within a community of inquirers. 8 To do research is thus to cosearch or to re-search along with the other searchers for knowledge within that particular field. If this communal discursive nature of research is ignored, then researchers run the risk of re-inventing the wheel over and over again.

What is thus required from business ethics researchers is to deliberately anchor their research in existing theory and also to reflect deliberately on

7 This is exactly what is lamented in the field of business ethics by Crane (1999:239), Robertson (1993:589) and Randall and Gibson (1990:461).

8 See Collier (1997) on research-as-dialogue in the German-speaking world and her doubts on whether it could work well outside that context. 
the implications of their findings for the theories from which their research originates. Only in this way can there be hope for the expansion of the theoretical basis of the field of business ethics. Finding a mutually stimulating relationship between theory and research practice is therefore vitally important, not only for expanding the theoretical base of the field of business ethics, but also for improving the quality of research and the academic stature of it.

\section{Conclusion}

This article demonstrated that business ethics is plagued by a number of recurring problems related to the ontology, methodology and strategy of research in this field of study, as well as by an unsatisfactory relation between research practice and theory building. These ailments are all curable. Guidelines were proposed for appropriate and relevant ontological assumptions, strategies and methodologies in research, as well as for the role that theory has to play in research. Adherence to these guidelines can contribute towards improving the quality of research in business ethics.

This presupposes that business ethics researchers have to be aware of these issues. But it is not only on the level of individual researchers that there should be increased awareness about research. It needs to be extended to the community of researchers, where ongoing reflection on trends and developments in research within this field should be kindled and kept alive. Meta-research, i.e. research on and analysis of the research that has already been done in the field of business ethics, can be a powerful stimulus to keep this process of reflection among researchers alive.

\section{Bibliography}

ARISTOTLE. 1976. The Nicomachean ethics. (Translated and introduced by J.A.K. Thomson.) Middlesex : Penguin

BAIN, W.A. 1995. Ethical problems in ethics research. Business Ethics: A European Review, 4(1):13-16.

BISHOP, J.D. 1991. The moral responsibility of corporate executives for disasters. Journal of Business Ethics, 10:377-383.

BRIGLEY, S. 1995a. Business ethics in context: researching with case studies. Journal of Business Ethics, 14:219-226.

BRIGLEY, S. 1995b. Business ethics research: a cultural perspective. Business Ethics: A European Review, 4(1):17-23.

BOTES, A. 2000. Arguments in research reports. (In Rossouw, G.J., ed. Intellectual tools: skills for human sciences. Pretoria : Amabhuku. p. 180-187.)

CAMBRIDGE DICTIONARY OF PHILOSOPHY. 1995. Cambridge : Cambridge University Press.

COLLIER, J. 1995. Business ethics research: shaping the agenda. Business Ethics: A European Review, 4(1):6-12. 
COLLIER, J. 1997. Business ethics research as dialogue: a European perspective. Business Ethics: A European Review, 6(3):168-174.

COWTON, C.J. 1998. The use of secondary data in business ethics research. Journal of Business Ethics, 17(4):423-434.

COWTON, C. \& CRISP, R. 1998. Business ethics: perspectives on the practice of theory. Oxford : Oxford University Press.

CRANE, A. 1999. Are you ethical? Please tick yes or no on researching ethics in organisations. Journal of Business Ethics, 20(3):237-248.

FRENCH, P.A. 1979. The corporation as moral person. American Philosophical Quarterly, 3:207-215.

LADD, J. 1984. Corporate mythology and individual responsibility. The International Journal of Applied Philosophy, 2:1-21.

MACINTYRE, A. 1985. After virtue: a study in moral theory. London : Duckworth.

MCDONALD, G.M. \& DONLEAVY, G.D. 1995. Objections to the teaching of business ethics. Journal of Business Ethics, 14(11):839-853.

MEYERS, C. 1993. The corporation, its members, and moral accountability. (In White, T.I., ed. Business Ethics: a philosophical reader. New York : MacMillan. p. 261-269.)

NICHOLSON, N. 1994. Ethics in organisations: a framework for theory and research. Journal of Business Ethics, 13:581-596.

RANDALL, D.M. \& GIBSON, A.M. 1990. Methodology in business ethics research: a review and critical assessment. Journal of Business Ethics, 9:457-471.

ROBERTSON, D.C. 1993. Empiricism in business ethics: suggested research directions. Journal of Business Ethics, 12:585-599.

VYAKARNAM, S. 1995. Focus groups: are they viable in ethics research? Business Ethics: A European Review, 4(1):24-29.

WERHANE, P. 1999. Moral imagination and management decision-making. Oxford : Oxford University Press.

\section{Key concepts:}

business ethics; research methodology

research ontology; business ethics

research strategy; business ethics

theory and practice of business ethics

\section{Kernbegrippe:}

besigheidsetiek; navorsingsmetodologie

navorsingsontologie; besigheidsetiek

navorsingstrategie; besigheidsetiek

teorie en praktyk; besigheidsetiek 